This is the final peer-reviewed accepted manuscript of:

R. Verdone and S. Mignardi, "Joint Aerial-Terrestrial Resource Management in UAVAided Mobile Radio Networks," in IEEE Network, vol. 32, no. 5, pp. 70-75, September/October 2018

The final published version is available online at:

http://dx.doi.org/10.1109/MNET.2018.1800036

Rights / License:

The terms and conditions for the reuse of this version of the manuscript are specified in the publishing policy. For all terms of use and more information see the publisher's website.

This item was downloaded from IRIS Università di Bologna (https://cris.unibo.it/)

When citing, please refer to the published version. 


\title{
Joint Aerial-Terrestrial Resource Management in UAV-Aided Mobile Radio Networks
}

\author{
Roberto Verdone, Silvia Mignardi
}

\begin{abstract}
This article addresses the issue of joint aerialterrestrial resource management in mobile radio networks supported by an unmanned aerial vehicle (UAV) operating as network node and discusses the potentials of a true integration between the terrestrial and the UAV components of the network. A simulation campaign shows that, by properly optimizing the system parameters related to the UAV flight, a single UAV can bring a significant improvement in network throughput for a wide service area. The use of a joint radio resource management (RRM) approach, where the UAV and terrestrial base stations operate in a coordinated manner, brings significant advantages with respect to different algorithms.
\end{abstract}

\section{INTRODUCTION}

In the next few years, research will address the long term evolution of the 5 th generation $(5 \mathrm{G})$ of mobile radio communications. It is expected that in dense areas, like urban environments, data traffic demand will soon increase by orders of magnitude [1]; this will be mostly due to video uploads and downloads requested by human users, and machines. In this context, the ability of the network to adapt efficiently to traffic demand evolutions in space and time, will be a key issue; this requires a degree of network flexibility much higher than in the past.

One way to achieve this flexibility, is by moving infrastructure nodes, reacting to the fast temporal/spatial variations of traffic demand. In this article (see Fig. 1) we assume that the terrestrial network is supported by an aerial component made of autonomous UAVs that carry on board base station (BS) equipment [2]. The main advantages of using UAVs in urban environments lie in:

- the ability to adapt the network infrastructure deployment to the current traffic demand,

- the ease to establish links with ground user equipments (UEs) having high Signal-to-Noise-Ratio (SNR),

- the possibility to improve service coverage and capacity where terrestrial base stations (TBSs) are ineffective.

UAVs might be carrying BSs with full capabilities, relay nodes, or the radio unit (RU) only of a BS. In the first two cases, UAVs will be equipped with a back-haul technology to ensure proper connectivity with the rest of the (terrestrial) network; in the latter, a front-haul will be needed to connect the RU to base band unit. In both cases the UAVs will require a high or very high capacity link with some radio ports deployed in the terrestrial component of the network. Owing

The authors are with the University of Bologna, Italy

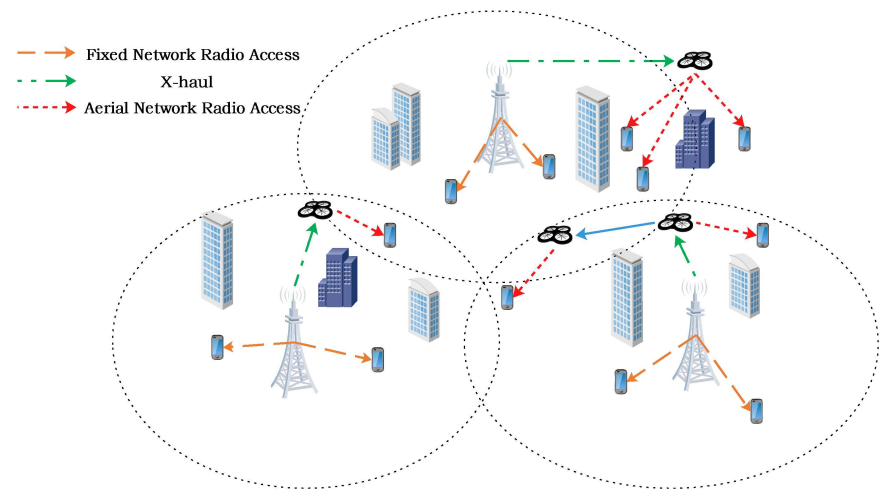

Fig. 1. An UAV-Aided Mobile Radio Network

to the ease of achieving a Line-of-Sight (LoS) link between a UAV and properly deployed radio terrestrial ports, the connectivity might be ensured by millimeter-wave or visible light technologies.

The first studies in the literature related to UAVs analyzed the air to ground (ATG) channel model, like [3]. The effects of LoS probability and the impact of the elevation angle between the UE on the ground and the UAV, were modeled.

For what concerns resource management, [4] investigates optimal resource allocation mechanisms for cluster heads (CHs) and UAV links with the goal of minimizing the transmission power in $\mathrm{CHs}$ while meeting the rate requirements of IoT devices. In [5], scheduling for UAV-aided networks is addressed, with the aim of achieving the maximum system performance in terms of encounter rate and energy efficiency. For what concerns UAVs deployment, Authors in [6] find dynamic 3D trajectories to connect all IoT devices at their activation time. They optimize jointly the transmission power of machine nodes, the overall energy for UAV mobility and the choice of each UAV next stop. In [7] considerations on the use of radio maps to drive UAVs are introduced, in order to exploit the effective environment dependent path loss. A discussion on the integration of UAVs in the next generation network is presented in [8]. Moreover, UAVs operate together with a cellular network in [9], where a delay-optimal cell association for ground users is proposed through optimal transport theory. In [10] UAVs mount a pico cell radio equipment and their efficiency in placement is compared to the static infrastructure counterpart. Furthermore, the Authors in [11] consider to offload traffic from the terrestrial network through a proactive deployment of drone-cells; it depends on three types of traffic and there is no interference between the ground network and 
the UAVs.

In [12], UAVs mount equipment of relay nodes not interfering with TBSs; the proposed model uses density and cost functions to calculate areas with higher demand and multiple UAVs are deployed depending on the beforehand expressed needs. First works studying the integration of terrestrial infrastructure and aerial platforms find an example in [13]. Authors analyze aspects such as radio access, backhaul links and coverage introduced by multiple drone-cells. However, they mainly focus on uplink machine type communication and do not consider a joint system resource management that includes trajectory planning.

In summary, some works in the literature already address the potential of UAV-aided mobile radio networks. Most of them consider the optimal placement of UAVs over the area to be served, in static scenarios where neither UAVs nor ground users move. In almost no case the issue of RRM is considered.

On the contrary, we focus here on RRM issues in a dynamic environment where the offered traffic evolves and UAVs fly at given speed. We believe that the integration of the aerial component in the context of the $5 \mathrm{G}$ network architecture, and the options for exploitation of a common pool of radio resources, are topics of potentially paramount relevance, not extensively addressed yet. In particular, in this article we study the delivery of high throughput services to users on the ground with non stringent delay constraints. Indeed, the advantages of using UAVs in urban environments are constrained by their limited speed and the time to reach users to offer their services. It is worth noting that the real-time identification of the optimal route of the UAVs, is a problem that should be addressed jointly with RRM. In fact, the UAV position, being a degree of freedom, can be seen as an additional dimension in the resource pool to be administered by L2 and L3 radio resource assignment algorithms. Results based on dynamic simulations are shown to support the discussion.

This work is organized as follows. After introducing the main achievements in scientific literature, we illustrate the network model and the urban 5G scenario considered. We propose a feasible approach for the system management and UAV's trajectory selection. Then, we analyze the RRM issues, focus of this work. In conclusion, numerical results and final discussion are presented.

\section{Network Model}

In this article, we refer to a scenario that will highlight the potential performance improvements achievable through integration of the aerial and terrestrial network components.

\section{Network Architecture}

5G network architectures will rely on approaches based on SDN (Software Defined Networking). An Orchestrator will be responsible, among other, of the global management of network resources; SDN controllers will locally manage resources made available by the physical network. We assume (see Fig. 2) that the mobile architecture network orchestrator (MANO) will include an interface towards a UAV network controller (UANC) that will be responsible for both managing the radio resources assigned by $\mathrm{UAV}_{\mathrm{s}}$, and defining their missions and trajectories. Through this interface, the UANC will be aware of all information related to the ground user traffic demand, and how the TBSs are serving it. In particular, a common assumption made in scientific literature is that the UAVs are aware of the positions of the ground users they have to serve; moreover, we can assume that the UANC has real-time information on the performance indicators and the resources exploited in all links between TBSs and UEs. Furthermore, the MANO, being able to track user locations, can decide to issue an handover for users between a UAV, under the UANC control, and the TBSs. In fact, the RRM algorithms perform re-assignment of all radio resources at each time step.

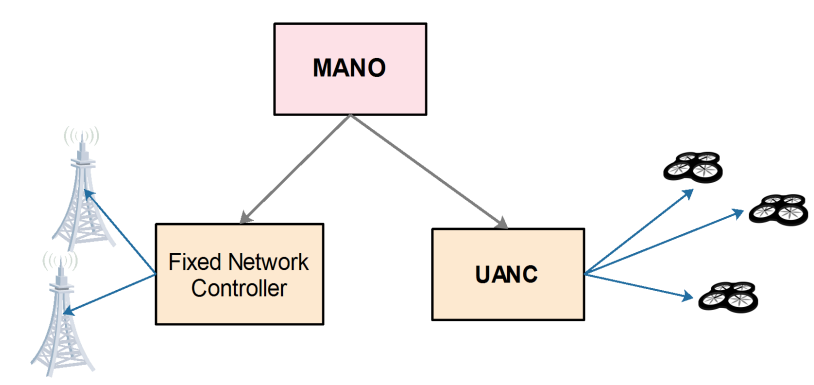

Fig. 2. Reference network architecture.

\section{Services of Interest}

UAVs fly with limited speed. Services with stringent delay requirements cannot benefit from the aerial component of the network. For this reason, the scientific literature has mainly considered two (broad) categories of delay tolerant applications so far: video streaming, and machine-to-machine type traffic. In the former case, the requirements set by the application include a maximum waiting time (normally in the order of seconds) before the uplink/downlink streaming starts, and a minimum throughput. In the latter, the application is often delay tolerant and requires the entire data stream to be transferred within a time window which can be very large. These traffic types are compatible with a service offered by UAVs. It is worth noting that in current (and future) mobile radio networks most of the traffic generated by users is in fact (expected to be) of video streaming type.

In our scenario, delay tolerance has a maximum of 24 seconds and the video download size is of $25 \mathrm{MB}$. For the sake of simplicity, we scale $5 \mathrm{G}$ downlink requirements to serve UEs (see TS 22.261 of $3 \mathrm{GPP}$ ) to a minimum of $10 \mathrm{Mb} / \mathrm{s}$ of throughput, to reach the same quality of service at both TBSs and UAV.

As a consequence, sum throughput, $S$, can be considered as a suitable key performance indicator to assess the benefits introduced by the aerial component of the network. $S$ is the sum over all UEs of the throughput perceived by each of them: it is estimated through the Shannon formula. 


\section{Traffic Area}

Most researchers are interested in urban environments, where the spatial/time variations of traffic can be significant and traffic volumes are large. This is the ideal scenario for an UAV-aided network. It may include macrocells, microcells and small cells. Some of the macrocell BSs, located on rooftops or towers, act as UAV homes. UAVs are usually parked in any of them (where their batteries can be recharged), moving only when required by the traffic demand, and flying along optimized trajectories defined according to network needs. It is also worth noting that UAVs can be part of daily network operation depending on their mechanical characteristics and weather conditions. Since we are considering a future $5 \mathrm{G}$ system, we can assume an improved UAV technology. Therefore, we consider that by the time this kind of network is deployed, the UAVs mechanics will be able to handle light-wind and light-rain. However, in those situations in which the UAV can not handle bad weather conditions, the UANC must call back UAVs to their recharge stations.

In this urban context, UAVs will be required to serve those UEs that cannot be served efficiently by the TBSs (we will denote them as unsatisfied users). They belong to three categories:

- UEs served with an insufficient amount of radio resources by the TBSs,

- UEs whose links have small values of SNR,

- UEs whose links have low SIR (Signal-to-InterferenceRatio).

In most cases, such users tend to be confined at the cell edges where SNR and SIR are normally smaller. So, the spatial distribution of candidate UEs will be far from uniform and UAV trajectories will privilege cell edges.

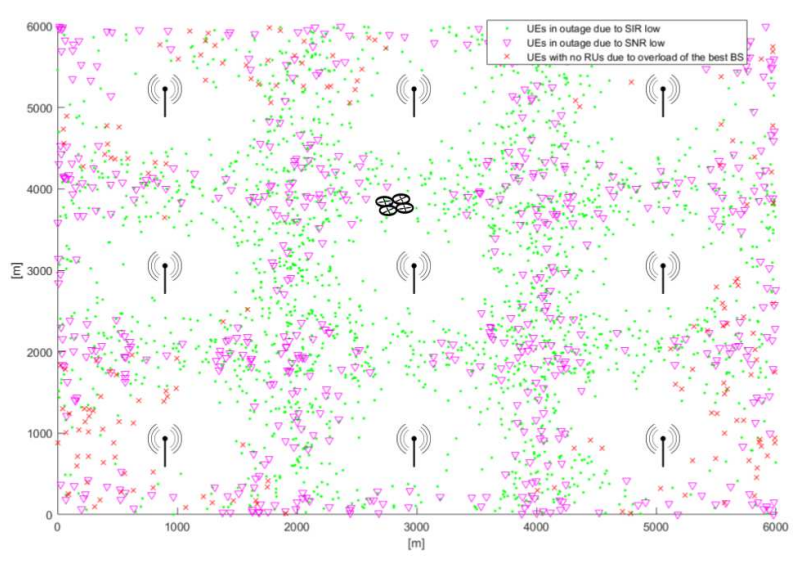

Fig. 3. Reference urban scenario in a simulation snapshot: unserved users are plotted.

In our simulations, we consider an $L$ by $L$ square urban area, with nine TBS sites deployed on a regular square grid, and four TBSs per site (see Fig. 3). So, each cell has an ideal area of $L^{2} / 36$ square meters.

\section{Physical Layer}

From the viewpoint of the air interface implemented on the aerial and terrestrial BSs, we refer to the LTE standard, from Release 8. This choice is made to assess the network model, but has no relevant impact for our final analysis. As for the frequencies used, we consider as a reference the $3.6 \mathrm{GHz}$ band, candidate for $5 \mathrm{G}$ services in cities.

Most papers assume the UAVs are equipped with a fixed directional antenna (or fixed coverage range) pointed towards the ground, with an angle of aperture of $\alpha$ degrees (see e.g. $[12,13])$. In the most simplistic case, assuming no radiation beyond this angle and constant antenna gain, the footprint on the ground (that is, the area covered by the BS carried by the UAV) is a circle of radius $r=h \cdot \tan (\alpha / 2)$, where $h$ is the UAV height. It is worth noting that, the larger is the UAV height, the larger is the footprint and the number of users that can be served by the UAV; however, the potential interference generated on other users on the ground, is also larger. Fixing $h$, the larger is $\alpha$, the larger is the footprint and the smaller is the antenna gain: some papers assume that the UAV antenna gain depends on $\alpha$ as $G_{\alpha}=29000 / \alpha^{2}$. We add to such gain 3 $\mathrm{dB}$ to account for a minimum level of directivity even for large $\alpha$. It is worth noting that the antenna directivity depends on physical aspects, like the size of the antenna and its shape, that for UAVs are closely connected to the specific type of flying platform used. It is out of the scope of this paper to account for such physical aspects. The analysis on the behaviour of $h$ and $\alpha$ do not change in a real case when channel fluctuations give a non-circular footprint shape [14]. Also, final simulations run with a more realistic channel.

\section{Radio Channel}

TBSs serve ground users through a channel affected by the usual impairments of urban environments: fading and shadowing, superimposed to the power loss of free space; typically, power loss depends on distance through an exponent between 3 and 4 , as it can be derived by the Okumura-Hata model.

On the opposite, the UAVs, owing to their position at considerable height (typical values are in the range 50-200 $\mathrm{m})$, can serve users on the ground through a LoS link, with power loss exponent equal to 2 and channel fluctuations kept to a minimum. Most papers in scientific literature use an ATG channel model (see e.g. [6, 9]) that describes the radio propagation through a two-state model: LoS and Non-LoS states are considered. The probability of $\operatorname{LoS}, P$, depends on the angle between the ground, and the line connecting the UE and the UAV. The larger is the angle, the closer to one is $P$ [3]. In the remainder of the article, the power loss is computed using the ATG model from [3].

\section{UAV TRAJECTORY DESIGN}

As shown previously, few papers address the optimal design of UAV paths. The reason lies in the complexity of the topic; in fact, the dynamic trajectory of UAVs should be defined according to a number of factors: the position of candidate 
UEs, their traffic requirements, the energy available on the $\mathrm{UAV}$, and so on. On the opposite, the optimal static placement of a UAV can be more easily computed, once the UE positions are known, accounting for the SNRs of all links established.

In our simulations we refer to the approach used in [2], that accounts for many of the factors that affect the scenario.

Assume an UAV is in position $Q(x, y, h)$ at a given instant $t$, when a new direction of flight has to be chosen. The MANO provides the UANC with the updated information on traffic demand, and radio resources assigned by TBSs. Based on this information:

1. the UANC groups the UEs that are unsatisfied according to a clusterization algorithm that generates $K$ clusters [2];

2. for each cluster $(i=1, \ldots, K)$, its centroid is computed starting from the knowledge of UE positions;

3. for each centroid $(i=1, \ldots, K)$, a cost function, $C_{i}$, is computed that accounts for all desired factors, including the centroid distance, $d_{i}$, from $Q$;

4. the centroid with the smallest cost function is identified (its distance from the current position is denoted as $d_{c}$ );

5. the UAV starts flying in the direction of the chosen centroid and keeps the same direction till when the centroid is reached, after a time $d_{c} / v$ where $v$ is the UAV speed;

6. during its flight, the UAV serves all UEs encountered, that would be unsatisfied otherwise.

The main idea behind this approach is that a UAV should fly in the direction where several users might be served; this is the reason for using clusters. Moreover, through $C_{i}$ a number of factors can be included. In this article, we consider the cost function defined in [14], reported below in simplified form:

$$
C_{i}=\left[\frac{d_{i}}{F_{1}}+\frac{\delta_{i}}{F_{2}}\right] \cdot\left[\frac{E_{i}}{F_{3}}\right] \cdot\left[\frac{1}{S_{i}}\right] \cdot F
$$

where:

- $\delta_{i}$ is the mean distance between UEs in cluster $i$, and its centroid (it measures the cluster compactness and is smaller for more compact clusters),

- $E_{i}$ is the energy consumed to reach the $i$-th centroid, computed as in [14, 15],

- $S_{i}$ is the sum throughput approximately estimated for underlying users when the UAV is at the $i$-th centroid,

- $F$ is a factor accounting for spatial fairness, avoiding sudden U-turns (see [14]),

- $F_{1}, F_{2}, F_{3}$ are normalizing factors (see [14]).

This approach will determine a trajectory made of a sequence of segments of different length. They are travelled by the UAV at constant speed $v$, and height from the ground $h$. Indeed, these two parameters should be subject to optimization (in combination with the choice of the antenna aperture angle $\alpha$ ), as they will significantly affect network performance. This article will highlight their role through the numerical results shown later. Finally, note that the factor $F_{3}$ accounts the fact that a UAV spends energy and has a limited lifetime. However, the scenario is simulated over an operation time of 30 minutes: professional UAVs having the same endurance already exist, and it is expected that in the coming years the technology available for UAVs will still improve.

In the case of multiple UAVs in the scenario, the cost function should include some factor oriented at avoiding collisions and towards an overall performance maximization. In this article, for the sake of brevity, we discuss the singleUAV case.

\section{RADio RESOURCE MANAGEMENT}

From the viewpoint of the radio resource pool used by the aerial network component to serve the ground users, there are several options.

\section{Separate Frequency Bands}

The simplest approach is to assume that UAVs serve the UEs using a different frequency band with respect to the TBSs. Of course, this excludes the presence of interference. On the other hand, the overall spectral network efficiency is not optimized. Most papers in the scientific literature implicitly consider this option (see e.g. $[11,12]$ ). We are not interested here in this simplistic scenario.

\section{Separate RRM Over the Same Frequency Band}

Let us assume the TBSs and UAVs use the same Radio Resource Unit (RRU) pool. The UANC is made aware of the RRU set used by any TBS to serve any UE both for the uplink and downlink. The UAV, while serving ground users, should choose the set of RRUs that will minimize or avoid any interference with the underlying terrestrial network.

It is worth noting that this approach is not based on a joint management of RRUs; the UAV assigns RRUs in order to keep interference under control, while leaving to the terrestrial network the power to choose freely what resources to assign in any link with the TBSs. In other words, the assignment of RRUs happens first at TBSs, then on the UAV.

Let us consider downlink streams first. The solution proposed in [14] represents the optimal choice under this scenario; assuming that the UAV determines a footprint whose area depends on $h$ and $\alpha$ as described earlier, the UAV will use at any instant only those RRUs that are unused by the UEs contained in its current footprint (known by the UANC). Under the assumption of an ideally directive antenna, the UAV transmission will have no impact on any other ground receiver outside its footprint; inside it, no UE is using the RRUs used by the UAV. Interference is neglected, while in fact the UAV is (re-)using some of the RRUs used by the terrestrial cell. In such situation, there is no negative contribution to the overall network throughput, while there might be positive contributions as long as some RRUs are available for serving unsatisfied UEs in the footprint. The larger the UAV footprint (whose area is $\pi r^{2}$ ), the larger the potential set of UEs to be served, while the number of available RRUs will be small. Clearly, there is an optimal footprint value to be found as tradeoff. 
Let us now consider the uplink and the same RRM strategy as above. For the UE-UAV link, there is no interference generated by UEs, because the UAV assigns orthogonally to its served UEs only RRUs unused in the footprint, and other uplink sources outside it do not contribute with received power at the UAV. However, the transmitted signals generated by UEs served by the UAV, might interfere at the TBSs with other UE transmissions in the cell; this effect can be minimized by using a reduced level of transmit power at the UEs served by the UAVs, and because of the high chance that they will be located at cell edge.

\section{Joint RRM}

Let us now assume that RRM is performed simultaneously and jointly at the UAV and TBSs. For any UE that can be served by both the terrestrial network and the UAV, the optimal choice is made. The advantage in this case with respect to the previous one is that there might be situations where a UE in the footprint of the UAV will be served with much higher SNR by the UAV, even though it might have been also served (but with lower throughput) by the TBS. An overall increase in terms of sum throughput is expected in this case.

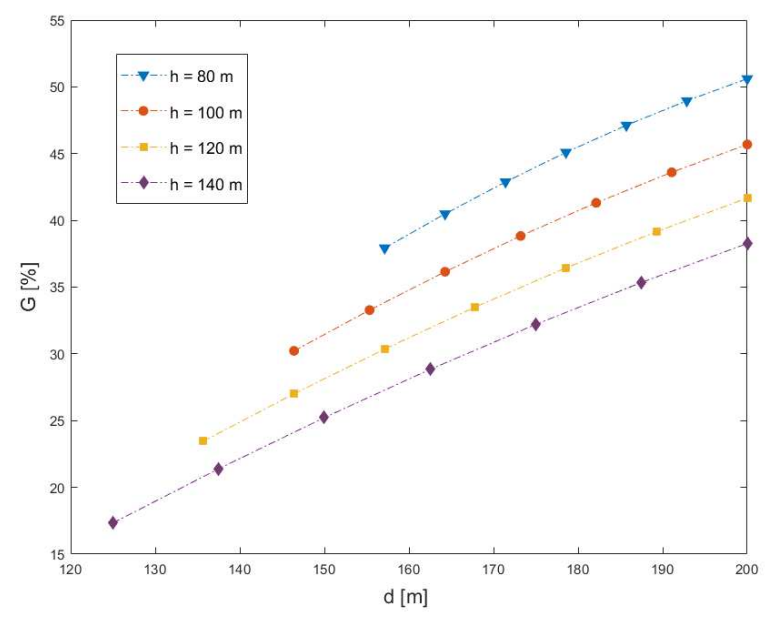

Fig. 4. Average throughput gain using the UAV instead of the TBS, as a function of the UE distance $d ; R=200 \mathrm{~m} ; \alpha=30$ degrees; $v=10 \mathrm{~m} / \mathrm{s}$; transmitted powers at TBS $P_{t(T B S)}=40 \mathrm{dBm}$ and UAV $P_{t(U A V)}=20$ $\mathrm{dBm}$; transmission gains at TBS $G_{t(T B S)}=100$ and UAV $G_{t(U A V)}=60$; propagation exponent for aerial links $\beta_{A T G}=2$.

To provide an estimation of such advantage, let us consider a TBS serving a macrocell of radius $R=200 \mathrm{~m}$, and a UAV covering in its trajectory a circular area of radius $r$ internal to the macrocell, adjacent to its border. Assume free space conditions for the UAV-UE link, while the TBS serves the UE at distance $d$ with a propagation exponent equal to 3.5 typical of urban environments. $P$ is set to one for the sake of simplicity. The SNR can be computed for the two links, and the Shannon capacities compared. Figure 4 shows the percentage gain, $G$, in terms of link throughput, using the UAV instead of the TBS to serve the UE, for all distances covered by the UAV ( $d$ ranging from $R-2 r$ to $R$ ). The gain is large, and would be much larger for larger values of $R$.

\section{NUMERICAL RESULTS}

We now refer to results obtained through the LTE-like dynamic simulator described in [14]. The simulated area has side $L=6000 \mathrm{~m}$. We average all results over 10 simulation runs, each considering 30 minutes of UAV flight.

Let us first analyze the impact on performance of the system parameters that determine the footprint size: $h$ and $\alpha$. Then, we consider the RRM strategy.

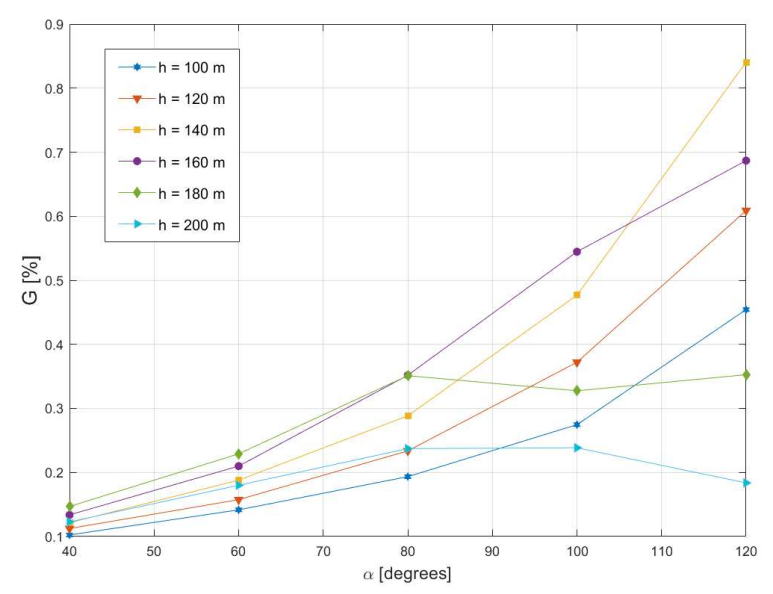

Fig. 5. Average throughput gain as a function of $\alpha$.

Figure 5 shows the throughput gain, $G$, in percentage, for different radiation angles $\alpha$. The speed $v$ is constant, its value equal to $10 \mathrm{~m} / \mathrm{s}$. A number of curves are obtained by varying the UAV height $h$. Every curve has an important antenna dependent effect: a maximum in throughput gain for an UAV height value. In fact, small altitude values generate small footprints and less users served. In contrast, increased heights give less RRU availability and therefore the UAV provides service to less users: larger coverage results in higher offered traffic and possibly lack of resources. Thus, a maximum in performance is obtained when a trade-off between coverage and RRU availability is reached; $h=140 \mathrm{~m}$ and large values of $\alpha$ seem to represent the optimal choice. We limit the analysis to $\alpha=120$ degrees because the adopted ATG channel model does not allow consideration of larger aperture angles [3].

Figure 6 highlights the effect of the previously mentioned joint RRM technique, with fixed height $h=140 \mathrm{~m}$. Two curves compare different throughput gains at varying radiation angles $\alpha$. The trend clearly shows that for each chosen $\alpha$, the joint RRM algorithm introduces a notable performance enhancement with respect to the separate RRM case.

\section{CONCLUSIONS}

This paper discusses the potential advantages of the integration between the terrestrial and aerial components of an 


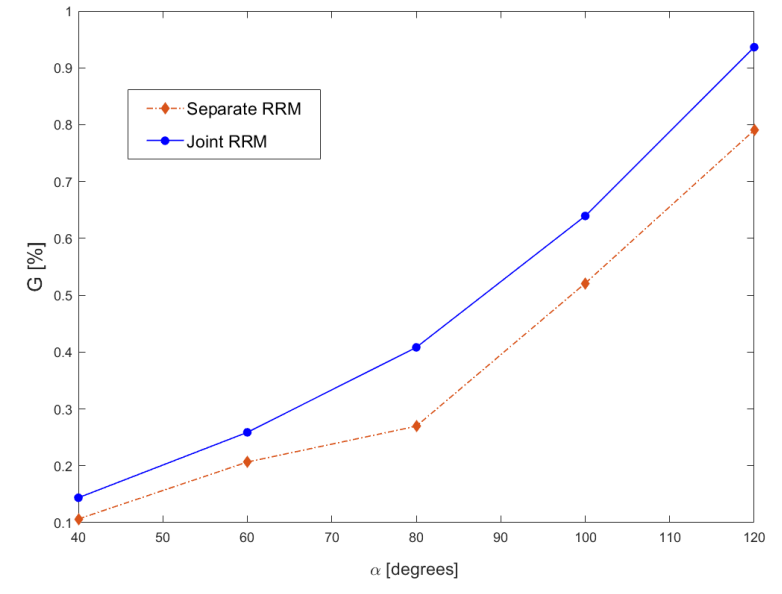

Fig. 6. Comparison of average throughput gains with separate and joint RRM as a function of $\alpha$.

UAV-aided mobile radio network, from the view point of RRM. Simulation results show that, by applying the discussed joint RRM strategy, a single aerial platform can bring an improvement in terms of network throughput of up to 1 percent. Though this value appears to be low, it has to be compared with the scenario size. In fact, we are considering a single UAV operation in a network of 9 TBS sites. Therefore, it becomes important to optimize the UAV use for costs and money saving. Moreover, it can pave the way to multiple UAV usage, which can bring to much larger performance increase.

In particular, we show that by using a joint RRM approach, where the UAV and the terrestrial base stations schedule the assignment of radio resources in a coordinated way, notable advantages are found with respect to the implementation of separate RRM algorithms.

However, there is one further step of potential improvement not discussed so far. As mentioned in the introduction, the design of the UAV trajectory might be included among the set of degrees of freedom used by the (joint) RRM strategies. Indeed, the UAV position at any instant represents a resource to be optimally used in order to achieve the target of an higher network throughput. In this paper, the choice of UAV trajectory is based on a number of factors; however, it does not account for the number of radio resources that the UAV will be allowed to exploit in the region it is going to visit. The inclusion of this consideration in the cost function used to choose the next cluster, might bring to further improvements. In other words, the (joint) RRM algorithms running at L3 of the protocol stack in the control plane, should also define the trajectory; inputs needed to define this path and their time evolution, are compatible with usual RRM algorithms.

This paper shows that a mobile radio network can provide better performance to its users when supported by an aerial infrastructure component. This is mainly due to the ideal channel conditions of a UAV-UE link, and the possibility to move the UAV according to traffic needs. An alternative to the use of UAVs is the integration of satellites in the terrestrial network. The same technologies that in this work are assumed to be applied to the UAV network component, might be used on the satellite layer. However, even at low orbits, satellites suffer from much higher propagation delays than UAVs flying at a height of few hundred meters. Moreover, the footprint of a UAV can be optimised to network needs, to reduce interference effects, while this can be more complex from a satellite. Nonetheless, UAVs suffer from some basic limitations; the most important stands in the complexity of managing their stability and flight under heavy weather conditions. For this reason, an extension to the concept developed in this paper might include integration of satellites in the overall architecture.

Finally, let us shortly discuss the role of antenna beamforming, not considered in this paper. Through the RRM policies mentioned above, the UAV and TBSs do not interfere each other in the downlink. So, the implementation of antenna beamforming at the UAV might introduce advantages in terms of throughput due to higher antenna gains, but not of interference avoidance. On the opposite, beamforming at the UE would improve performance for the uplink case. Such advantage is left for further studies.

\section{REFERENCES}

[1] CISCO, "CISCO visual networking index: Global mobile data traffic forecast update 2016-2021," C. V. Forecast, Sept 2017.

[2] S. Mignardi and R. Verdone, "On the performance improvement of a cellular network supported by an unmanned aerial base station," in Teletraffic Congress (ITC 29), 2017 29th International, vol. 2. IEEE, 2017, pp. 7-12.

[3] A. Al-Hourani, S. Kandeepan, and A. Jamalipour, "Modeling air-to-ground path loss for low altitude platforms in urban environments," in 2014 IEEE Global Communications Conference. IEEE, 2014, pp. 2898-2904.

[4] M. N. Soorki, M. Mozaffari, W. Saad, M. H. Manshaei, and H. Saidi, "Resource allocation for machineto-machine communications with unmanned aerial vehicles," in Globecom Workshops (GC Wkshps), 2016 IEEE. IEEE, 2016, pp. 1-6.

[5] S. Koulali, E. Sabir, T. Taleb, and M. Azizi, "A green strategic activity scheduling for UAV networks: A submodular game perspective," IEEE Communications Magazine, vol. 54, no. 5, pp. 58-64, 2016.

[6] M. Mozaffari, W. Saad, M. Bennis, and M. Debbah, "Mobile unmanned aerial vehicles (UAVs) for energy-efficient internet of things communications," IEEE Transactions on Wireless Communications, vol. 16, no. 11, pp. 75747589, Nov 2017.

[7] J. Chen, U. Yatnalli, and D. Gesbert, "Learning radio maps for UAV-aided wireless networks: A segmented regression approach," in Communications (ICC), 2017 IEEE International Conference on. IEEE, 2017, pp. $1-6$. 
[8] I. Bor-Yaliniz and H. Yanikomeroglu, "The new frontier in RAN heterogeneity: Multi-tier drone-cells," IEEE Communications Magazine, vol. 54, no. 11, pp. 48-55, 2016.

[9] M. Mozaffari, W. Saad, M. Bennis, and M. Debbah, "Optimal transport theory for cell association in UAVenabled cellular networks," IEEE Communications Letters, 2017.

[10] B. Galkin, J. Kibilda, and L. A. DaSilva, "Deployment of UAV-mounted access points according to spatial user locations in two-tier cellular networks," in Wireless Days (WD), 2016. IEEE, 2016, pp. 1-6.

[11] P. Yang, X. Cao, C. Yin, Z. Xiao, X. Xi, and D. Wu, "Proactive drone-cell deployment: Overload relief for a cellular network under flash crowd traffic," IEEE Transactions on Intelligent Transportation Systems, vol. 18, no. 10, pp. 2877-2892, Oct 2017.

[12] V. Sharma, M. Bennis, and R. Kumar, "UAV-assisted heterogeneous networks for capacity enhancement," IEEE Communications Letters, vol. 20, no. 6, pp. 1207-1210, 2016.

[13] Y. Li and L. Cai, "UAV-assisted dynamic coverage in a heterogeneous cellular system," IEEE Network, vol. 31, no. 4, pp. 56-61, July 2017.

[14] S. Mignardi, C. Buratti, and R. Verdone, "On the impact of radio channel over REM-aware UAV-aided mobile networks," in 22nd International ITG Workshop on Smart Antennas (WSA 2018).

[15] Y. Zeng and R. Zhang, "Energy-efficient UAV communication with trajectory optimization," IEEE Transactions on Wireless Communications, vol. 16, no. 6, pp. 37473760, 2017.

\section{BIOGRAPHIES}

Roberto VERDONe received his Ph.D. degree from the University of Bologna, where he is Full Professor in Telecommunications. He was involved in European projects like NEWCOM in 2004 as Vice-Director. He also participated to the FP7 NoE CRUISE. He coordinates activities of the University of Bologna within the ARTEMIS project eDiana and the FP7 project Wiserban. He was Director of the distributed laboratory EuWIn "European laboratory of Wireless communications for the future Internet", in the framework of the NoE NEWCOM, funded by FP7. His research activity is concerned with both infrastructure-based radio networks (Cellular and Mobile Radio Systems) and infrastructure-less radio networks (Ad Hoc and Wireless Sensor Networks, Vehicular Networks).

Silvia MignaRdi receveid her B.S and M.Sc. degrees at the University of Bologna in Dec. 2016. She is currently a first year PhD Student at the University of Bologna, Italy. She was also a collaborator in the IoT Startup IDESIO, working as a firmware designer. Her main research interest includes the analysis and integration in the current infrastructure of UAVAided Networks (or Moving Networks) where UAVs become flying network nodes. 